\title{
Microneedle array sensors based on carbon nanoparticle composites: interfacial chemistry and electroanalytical properties
}

\author{
Catherine Hegarty ${ }^{1, *}$ (D), Stephen McKillop ${ }^{1}$ (D), Ruairi J. McGlynn ${ }^{1}$ (D), Robert B. Smith ${ }^{2}$ (D), \\ Ashish Mathur ${ }^{3}$ (D), and James Davis ${ }^{1}$ (D) \\ ${ }^{1}$ School of Engineering, Ulster University, Jordanstown, Northern Ireland BT37 OQB, UK \\ ${ }^{2}$ Chemistry, School of Physical Sciences and Computing, University of Central Lancashire, Preston PR1 2HE, UK \\ ${ }^{3}$ Amity Institute of Nanotechnology, Amity University, Noida, Uttar Pradesh 201313, India
}

Received: 21 February 2019

Accepted: 16 April 2019

Published online:

30 April 2019

(C) The Author(s) 2019

\begin{abstract}
Conductive microneedle patches consisting of carbon nanoparticles embedded in a polystyrene matrix have been prepared using micro-moulding techniques. The interfacial properties of the structures before and after electrochemical etching have been characterised using X-ray photoelectron spectroscopy and contact angle. Anodisation of the needles leads to a significant increase in oxygen functionality and is shown to dramatically improve the electroanalytical capabilities of the microneedle array. The detection of uric acid in horse blood was used as a model system through which to assess the performance of the system. The composite approach is shown to lead to viable carbon-based sensors and can offer a rapid prototype option for the development of tailored microneedle systems.
\end{abstract}

\section{Introduction}

Microneedle (MN) patches have garnered extensive interest for the transdermal delivery of drugs and vaccines where the small dimensions of the needles penetrating the skin fail to trigger the dermal nerves and offer near painless injection [1-3]. A wide variety of approaches have been investigated in the design and construction of the microneedle masters but tend to revolve around the lithography/etching of resist layers on silicon wafers [4-6] or the CNC milling of metal blocks (typically aluminium [7-10]). Lim and colleagues have provided a critique of the approaches taken in microneedle [7, 11], but, irrespective of the process taken to acquire the master, the replication workhorse templates are almost invariably produced using PDMS [4, 7, 8, 10, 12, 13]. The high specification of the instruments needed to produce the master template was once a major hurdle and has often restricted availability of the PDMS replicate moulds to the host group. The increasing commercial availability of PDMS moulds covering a wide range of needle designs, however, provides greater opportunities through which new MN formulations can be

Address correspondence to E-mail: Hegarty-C19@ulster.ac.uk 
developed and targeted at different applications. The ability to manipulate the material composition of the MN patch has obvious advantages for therapeutic delivery applications, but it has also been shown to enable the production of $\mathrm{MN}$ arrays for sensing applications. A number of approaches have been taken in the development of electrochemical MN sensors for transdermal glucose monitoring but most rely on the deposition of $\mathrm{Au}$ or Pt onto preformed MN arrays [4, 6, 8, 14, 15].

The fabrication of MN arrays based on conductive composites from "off the shelf" PDMS moulds offers a potentially simpler and faster route to the prototyping of biosensors and one which avoids the instrumental overheads necessary for the preparation of master templates. The potential viability of the approach was initially demonstrated through the fabrication of conductive palladium/polycarbonate MN structures [16]. The development of carbonbased MN arrays, however, could yield a much more versatile system and, through exploiting a wealth of existing electroanalytical methods based on carbon electrodes, open up new avenues for transdermal sensing. In particular, the large overpotential required for hydrogen evolution at carbon could offer much wider potential ranges for electroanalytical investigations than those employing gold or platinum $[4,6,8,14,15]$. The surface chemistries of the exposed carbon nanoparticles could also offer catalytic capabilities through the presence of endogenous quinone functionalities [17]. Moreover, the presence of interfacial carboxylic acid groups could also serve as a simple anchor through which biomolecules (enzymes/antibodies) could be tethered through conventional carbodiimide coupling [18, 19]. A possible disadvantage, however, relates to the granular nature of the carbon particles which could compromise the mechanical integrity of the needles. The adoption of nanoparticles is critical in this aspect allowing greater incorporation and physical interaction with the polymeric binder of the composite. The aim of the present communication has been to investigate the development of conductive carbon microneedle arrays based on a carbon nanoparticlepolystyrene composite and to examine the modification of the interfacial surface functionalities to yield sensors with enhanced electroanalytical capabilities.

\section{Fabrication methodology}

Composite microneedles have previously been prepared through solvent casting a suspension of metallic particles in a polymer/cyclohexanone mixture into a pre-patterned silicone template [16]. The latter determines the number and dimensions of the needles in the resulting MN patch. Once the solvent has evaporated, a solid composite $\mathrm{MN}$ array remains in which the conductive particle is homogenously distributed within a polymer binder. In the present instance, the intention was to use carbon nanoparticles $(<100 \mathrm{~nm})$ as the conductive component and polystyrene as the binder with the distribution of the carbon enabling conduction from needle tip through the bulk to the base plate. The basic design is highlighted in Fig. 1 with electrical contact to the potentiostat achieved through connection to the back of the base plate. It can be expected that graphitic carbon is present at the interface and, although possessing a range of surface functionalities and defects, will consist of a largely basal plane structure as indicated in Fig. 1b. The latter presents a number of issues in that such structures can exhibit slow electron transfer kinetics towards biomolecular targets such as uric [20, 21]. Electrochemical anodisation is known to improve electroanalytical performance at solid carbon surfaces such as screen-printed carbon [21] and carbon fibre [17], but it is possible such procedures could be deleterious to the needle microstructures here.

The electroanalytical performance of the MN system was evaluated through examining the response to uric acid. The latter is a common biomarker in a range of clinical conditions, such as hypertension [22], kidney function [23], stroke [24] and cardiovascular disease $[25,26]$. It is little surprise therefore that there is an extensive literature base on its electrochemical detection and, with continuing advances in 2D carbon nanomaterials and other catalytic particles, continues to hold the interest of the diagnostics [27-31]. While the present investigation has sought to examine the material characteristics of the composite $\mathrm{MN}$ array, the response to urate within horse blood, as a model system, was critically assessed along with its potential translation to transdermal applications. 


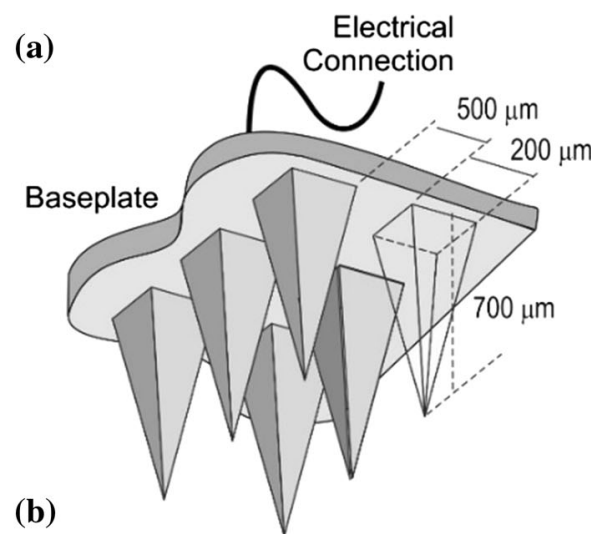

Carbon-Polystyrene Interfacial Carbon Microneedle

Nanoparticle

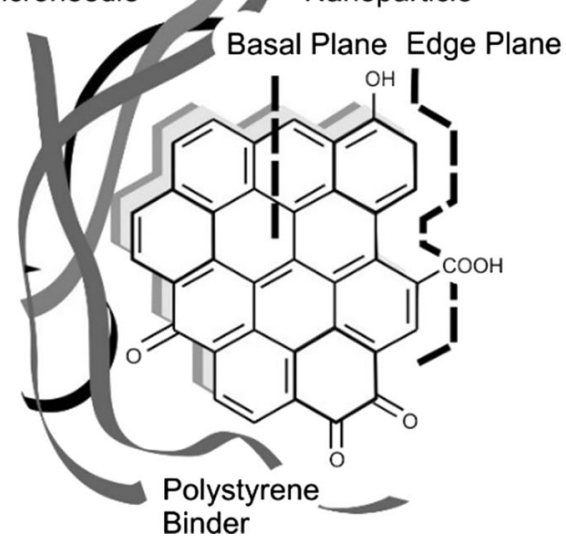

Figure 1 a Dimensions of microneedle array and $\mathbf{b}$ interfacial structure of carbon nanoparticles within the microneedle array.

\section{Experimental details}

\section{Materials and instrumentation}

Carbon nanopowder $(<100 \mathrm{~nm}$ ), polystyrene (MW 192000) and all associated laboratory chemicals were obtained from Sigma-Aldrich, were the highest grade available and were used without further purification. Britton Robinson buffers (acetic, boric, and phosphoric acids, each at a concentration of $0.04 \mathrm{M}$ and adjusted to the appropriate $\mathrm{pH}$ through the addition of sodium hydroxide) were used throughout unless otherwise specified. Defibrinated horse blood (without preservative) was obtained from Fischer Scientific (Thermo Scientific Oxoid 100ML) and used upon receipt. Microneedle moulds were obtained from Micropoint Technologies Pte Ltd (Singapore) and were pyramidal in format with 200 (base) $\times 500$ (pitch) $\times 700$ (height) micron dimensions covering a $10 \times 10$ needle array.

\section{Instrumentation}

Electrochemical analysis was carried out using a micro-Autolab (Type III) computer controlled potentiostat (Eco-Chemie) with a standard threeelectrode configuration in which the microneedle patch was used as the working electrode with platinum and a $\mathrm{Ag} \mid \mathrm{AgCl}$ half cell $(3 \mathrm{M} \mathrm{NaCl}, \mathrm{BAS}$ Technicol UK) acting as counter and reference, respectively. All measurements were taken at $22{ }^{\circ} \mathrm{C} \pm 2{ }^{\circ} \mathrm{C}$. Conductivity measurements were taken using a 2461 series SourceMeter ${ }^{\circledR}$ (Keithley) four-point probe. The conductivity of an unmodified carbon-polystyrene microneedle patch was found to be $1575.2 \mathrm{~S} / \mathrm{m} \pm 96 \mathrm{~S} / \mathrm{m}(N=5)$.

Static contact angle measurements were taken using a CAM 200 optical contact angle meter (KSV Instruments Ltd.). The sessile drop method was employed with deionised water $(5 \mu \mathrm{L})$ used as the probe liquid. Left and right contact angles were then calculated from the high-resolution CCD camera images using drop shape analysis.

A Kratos Axis Ultra DLD Spectrometer was used to quantify surface composition and acquire X-ray photoelectron spectroscopy (XPS) spectra. Spectra were analysed using monochromated $\mathrm{Al} \mathrm{K} \alpha$ X-rays $[h v=1486.6$ electron volts $(\mathrm{eV})]$ with typical operating parameters of $15 \mathrm{kV}$ and $10 \mathrm{~mA}(150 \mathrm{~W})$. During analysis, a hybrid lens mode was used (electrostatic and magnetic) with a $300 \mu \mathrm{m} \times 700 \mu \mathrm{m}$ analysis area and a take-off angle (TOA) of $90^{\circ}$ with respect to the sample surface. Wide energy survey scans (WESS) were collected across a range of -5 to $1200 \mathrm{eV}$ binding energy (BE), with a pass energy of $160 \mathrm{eV}$ and step size of $1 \mathrm{eV}$. High-resolution spectra were collected with a pass energy of $20 \mathrm{eV}$ with a $0.05 \mathrm{eV}$ step size, a scan width of $25 \mathrm{eV}$, a dwell time of $150 \mathrm{~ms}$ and at least 3 sweeps to reduce the signal noise. A Kratos charge neutraliser system with a filament current between 1.8 and $1.95 \mathrm{~A}$ and a charge balance of 3.3-3.6 V and a filament bias of $1.3 \mathrm{~V}$ was used for all samples. Charging effects on the $\mathrm{BE}$ positions were adjusted by setting the lowest $\mathrm{BE}$ for the $\mathrm{C} 1 \mathrm{~s}$ spectral envelope to $284.8 \mathrm{eV}$, which is commonly accepted as adventitious carbon surface contamination. Three measurements were taken per sample, with a Shirley background subtracted from each XPS spectra. The peak areas of the most intense spectral lines for each elemental species were used to determine the percentage atomic concentration. Peak 
fitting of high-resolution spectra was carried out using Casa XPS software.

\section{Preparation of microneedles}

Carbon nanoparticles were combined with polystyrene powder in a ratio of 1:1 by weight, dissolved in cyclohexanone and stirred until a homogenous solution was obtained (typically $2 \mathrm{~h}$ ). The solution was then cast into silicone microneedle templates, and a carbon fibre stub was placed into the base plate section to facilitate electrical connection. The templates were placed in a vacuum at $30{ }^{\circ} \mathrm{C}$, and once the pressure had increased to 1000 mbar, the air was released again. (This was to draw the homogenous solution to the tips of the silicone cast ensuring sharp microneedle production.) If required after vacuuming, the templates were topped up with more solution preferably overflowing the cast to allow for solvent evaporation. The solvent was left to evaporate at room temperature (over $48 \mathrm{~h}$ ), whereupon the needles could be removed from the patch. A typical silicone microneedle template $\left(\right.$ Micropoint ${ }^{\circledR}$ ) is shown in Fig. 2a along with a scanning electron micrograph detailing the morphology of the carbonpolystyrene microneedle array. The baseplate and non-needle surfaces were coated with enamel $(6 \mathrm{~h}$ drying period) to serve as a dielectric and define the geometric electrode area.

\section{Results}

Square wave voltammograms detailing the response of the unmodified carbon $\mathrm{MN}$ array towards uric acid in pH 7 buffer are shown in Fig. 3. A broad oxidation peak can be seen at $+0.6 \mathrm{~V}$ which increases with increasing urate concentration. In contrast, the response obtained at a carbon $\mathrm{MN}$ array which had been anodised for 5 min under similar conditions exhibits a sharp peak at $+0.3 \mathrm{~V}$ with a dramatically increased current response. The shift in the oxidation potential can be attributed to an increase edge plane sites (c.f. Fig. 1) and oxygen functionality that is known to improve electron transfer kinetics. The limit of detection was found to be $2.85 \mu \mathrm{M}$ (based on $3.3 S_{b} / m$ ) and is well within the range expected for UA within many biofluids. The response sensitivity was $361 \mu \mathrm{A} \mathrm{mM} \mathrm{m}^{-1} \mathrm{~cm}^{-2}$ and, while many literature reports fail to provide comparative data, of those that
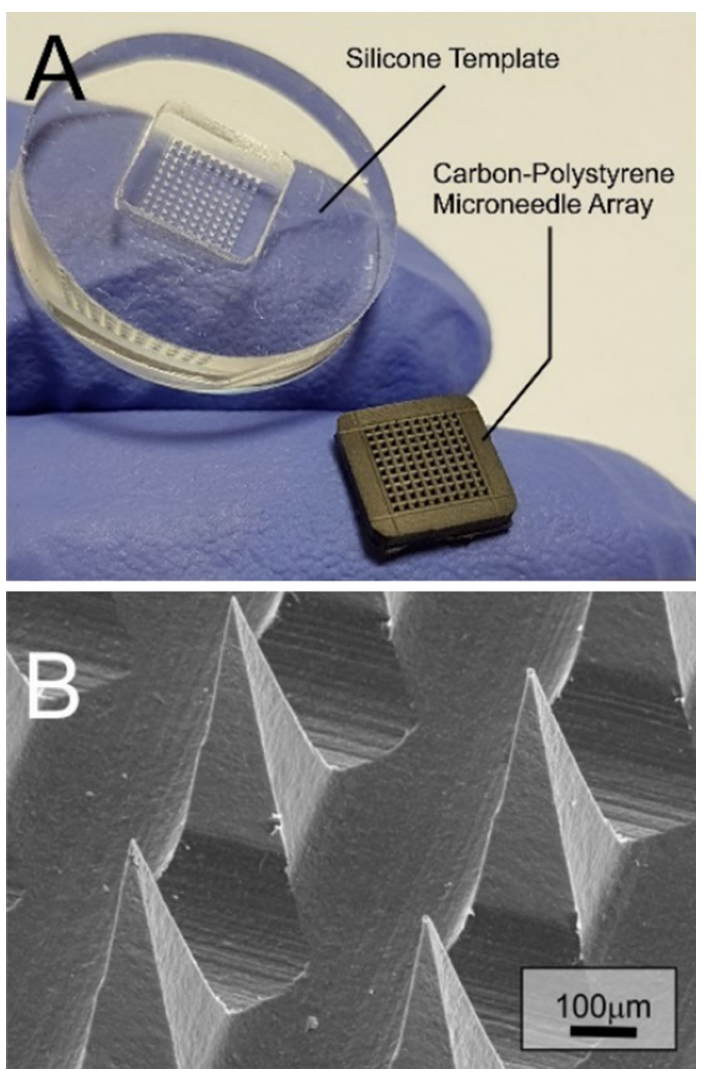

Figure 2 a Silicone template used for micro-moulding the $10 \times 10$ microneedle array. $\mathbf{b}$ Scanning electron micrograph of a carbon nanoparticle-polystyrene microneedle array obtained.

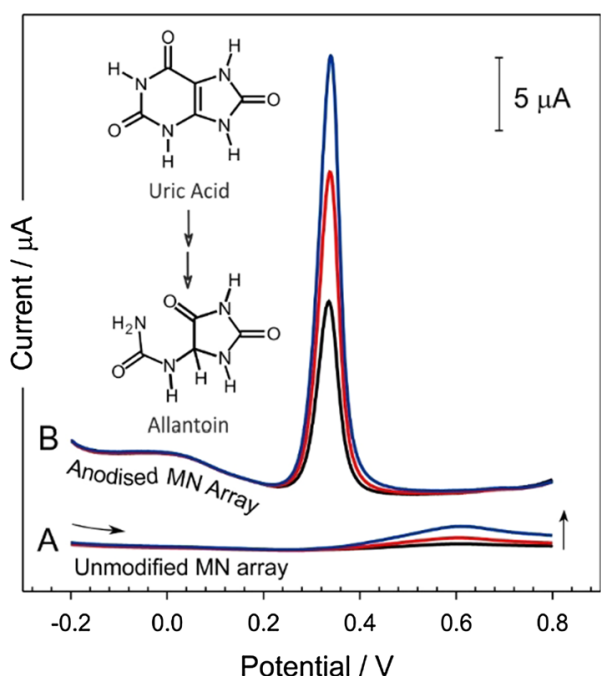

Figure 3 Square wave voltammograms detailing the response of a carbon nanoparticle-polystyrene microneedle array towards uric acid additions in $\mathrm{pH} 7$ buffer before (a) and after (b) electrochemical anodisation. 
do [32-34], the system here appears competitive without having to undergo the more complex surface modifications.

A detailed investigation of the carbon interface was conducted using high resolution $\mathrm{X}$-ray photoelectron spectroscopy. Spectra detailing changes in the C $1 \mathrm{~s}$ profile before and after $5 \mathrm{~min}$ of anodisation are compared in Fig. 4a, b, respectively. It can be seen from the XPS data that there is a significant increase in oxygen functionality at the carbon $\mathrm{MN}$ interface after anodisation and this stands in marked contrast to the simpler spectrum observed with the unmodified array. The interface is composed of carbon entrapped in a polystyrene matrix and, while both components will contribute to the $\mathrm{sp}^{2}$ signal, any reduction in magnitude of this signal can be attributed principally to the oxidation of the carbon. It
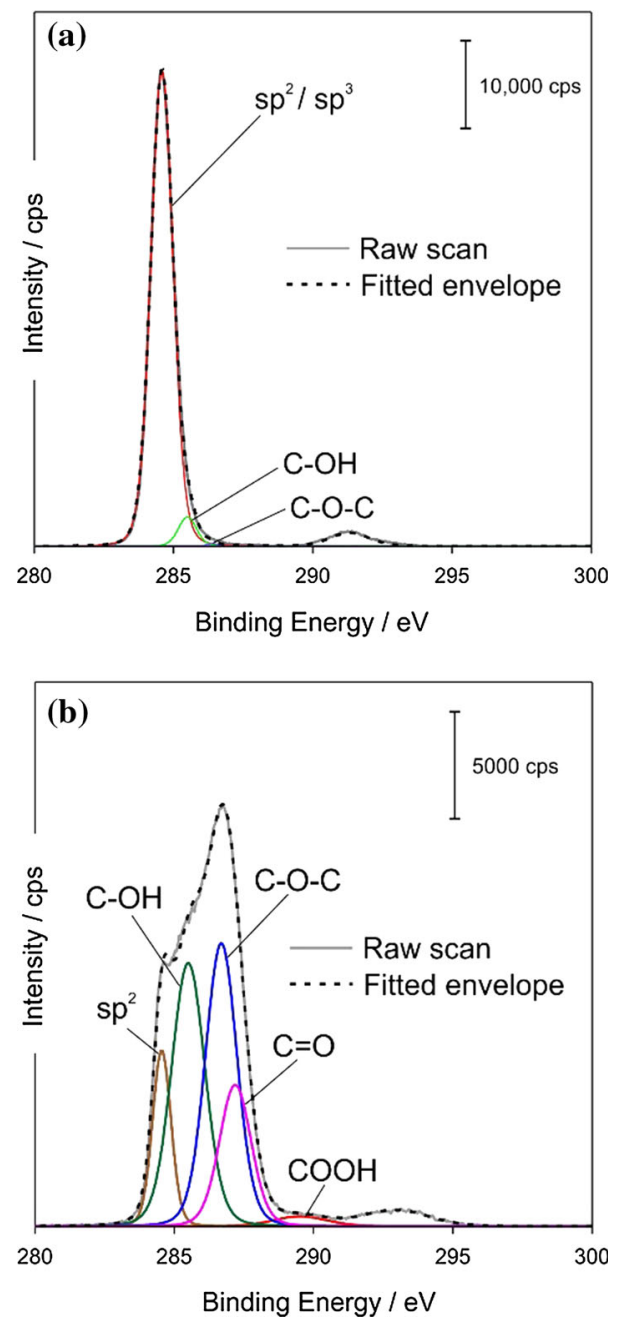

Figure 4 XPS analysis of a carbon-polystyrene microneedle array before (a) and after (b) electrochemical anodisation. should be noted that there will be $\mathrm{sp}^{2}$ and $\mathrm{sp}^{3}$ moieties at interface and it is not feasible to distinguish between the two under the present operating conditions.

The non conductive nature of the polystyrene and unreactive nature of its aromatic rings (and hence the large $\mathrm{sp}^{2}$ contribution) could be expected to change little during the anodisation process. A detailed breakdown of the \% composition for the various carbon-oxygen surface functionalities is highlighted in Table 1. The increased oxygen functionality is broadly consistent with previous studies by Anderson and co-workers who examined the anodic oxidation of carbon fibre [17].

Given the significant lack of oxygen functionality in the unanodised $\mathrm{MN}$ array, it would be expected to express considerable hydrophobicity. In contrast, the anodised MN array contains a multitude of polar groups and a high degree of intercalated water which should greatly improve surface wetting. The contact angle for single component polystyrene film formed from the solvent casting of the material used as the binder in the MN arrays onto a planar silicone sheet (devoid of ${ }^{* *}$ the microneedle features) was found to be $88.7^{\circ} \pm 0.9^{\circ}(N=6)$ which is consistent with previous studies of the polymer. The angles found with the unmodified carbon-polystyrene films and those that had been anodised yielded angles of $98.1^{\circ} \pm 0.81^{\circ}$ and $95.2^{\circ} \pm 2.9^{\circ}$, respectively. Thus, while there is a moderate increase in hydrophobicity associated with the inclusion of the carbon nanoparticles-the process of electrochemical anodisation does little to increase the hydrophilicity. The contact angle for the actual MN composites shows a further increase in hydrophobicity with the unmodified and anodised systems exhibiting angles of $115^{\circ} \pm 7.7^{\circ}$ and $108^{\circ} \pm 1.3^{\circ}$, respectively. Representative contact angle images highlighting the hydrophobicity of the MN system are shown in Fig. 5.

\section{Electroanalytical capability}

A preliminary assessment of the analytical viability of the anodised MN was conducted using defibrinated horse blood (HB) as the test matrix. The uric acid concentration within the HB was analysed using a standard addition protocol in which known amounts of a standard urate solution (typically $50 \mu \mathrm{L}$ aliquots of a $0.01 \mathrm{M}$ solution) were added directly to 
Table 1 Deconvoluted data highlighting the change in carbon-oxygen functionality pre and post anodisation of the $\mathrm{MN}$ array

\begin{tabular}{llll}
\hline & Peak Position/eV & \multicolumn{2}{l}{ \% Composition $^{\mathrm{a}}$} \\
\cline { 3 - 4 } & & Unmodified MN & Anodised MN \\
\hline $\mathrm{C} 1 \mathrm{~s}$ & & & \\
$\mathrm{Sp}^{2}$ & 284.57 & 73.4 & 13.0 \\
$\mathrm{C}-\mathrm{OH}$ & 285.50 & 12.7 & 28.9 \\
$\mathrm{C}-\mathrm{O}-\mathrm{C}$ & 286.52 & 10.3 & 31.8 \\
$\mathrm{C}=\mathrm{O}$ & 287.50 & v.low & 19.3 \\
$\mathrm{COOH}$ & 289.13 & v.low & 2.5 \\
$\mathrm{Pi}$ to P & 292.11 & 3.7 & 4.5 \\
$\mathrm{O} 1 \mathrm{~s}$ & & & 1.4 \\
$\mathrm{C}=\mathrm{O}$ & 531.47 & 3.7 & 24.5 \\
$\mathrm{C}-\mathrm{O}$ & 532.47 & 59.1 & 24.1 \\
$\mathrm{C}-\mathrm{OH}$ & 533.80 & 19.4 & 50.0 \\
$\mathrm{Absorbed}$ water & 534.14 & 17.8 & \\
\hline
\end{tabular}

${ }^{\mathrm{a}}$ Mean values based on 3 replicate measurements
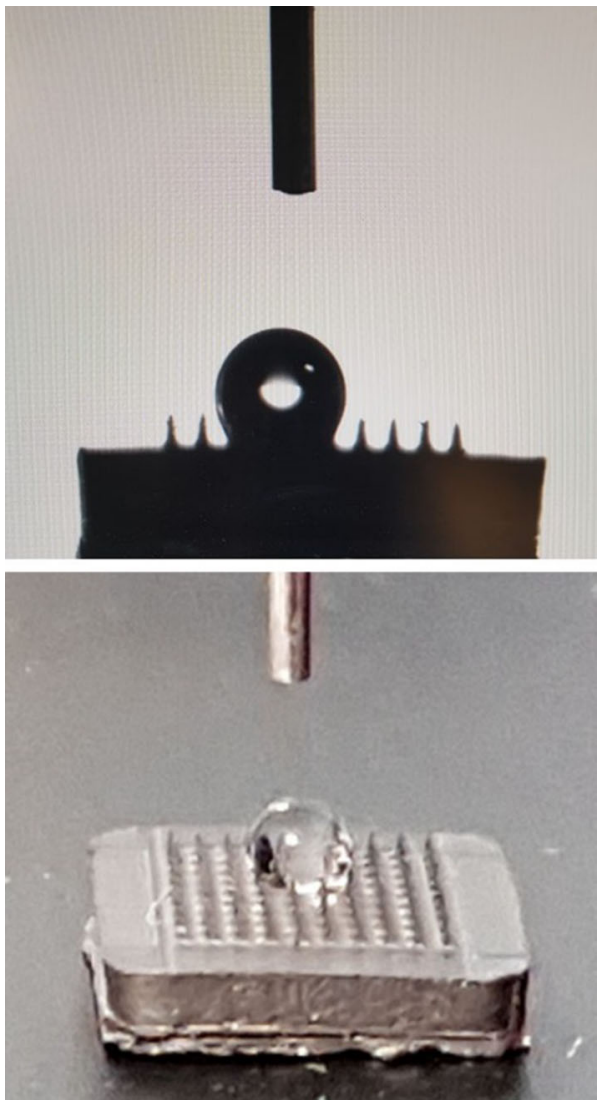

Figure 5 Contact angle measurement conducted on an unmodified carbon-polystyrene microneedle array without any electrochemical modification.

$10 \mathrm{~mL}$ of the sample. Square wave voltammograms detailing the response of the anodised $\mathrm{MN}$ to $\mathrm{HB}$ in the absence and presence of increasing additions of standard urate are detailed in Fig. 6. A distinct peak attributed to the oxidation of urate can be observed in the HB solution which increases in magnitude as the standard urate is added. The scan parameters are narrowed to 0 to $+0.5 \mathrm{~V}$ to minimise oxidation of other components within the sample which would otherwise foul the surface [21]. The reference range for urate in horse blood (plasma) is typically $30-250 \mu \mathrm{M}$ and varies depending on the relative health of the animal [35]. The electrochemical analysis of the defibrinated HB highlighted in Fig. 6 yielded $155 \mu \mathrm{M}$.

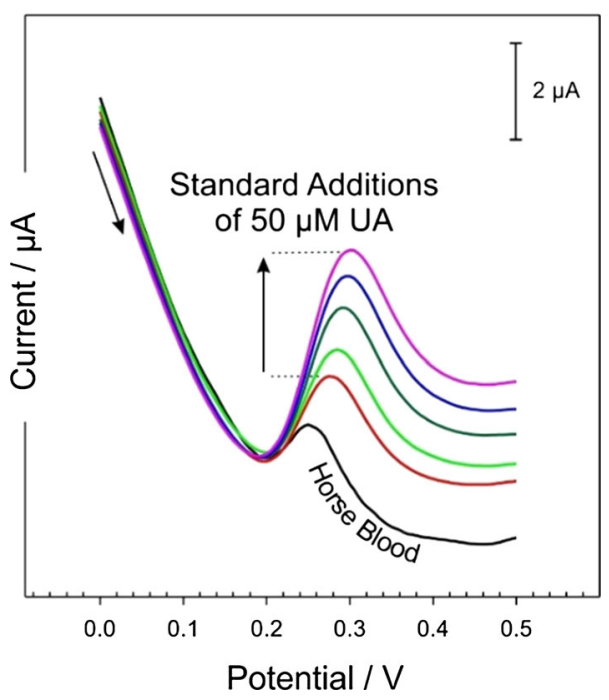

Figure 6 Square wave voltammograms detailing the response of an anodised $\mathrm{MN}$ array in $\mathrm{HB}$ before and after the addition of standard uric acid. 
A slight peak shift can be seen in Fig. 6 and can be attributed to the gradual fouling of the electrode surface. Similar issues have been reported by Phair and colleagues who found that screen-printed carbon electrodes operating within whole blood samples were susceptible to surface contamination by proteins and other macromolecular species [21]. While restricting the scan range minimises the effect of tyrosine and tryptophan oxidation leading to electrode passivation, the bare electrode remains susceptible to passive adsorption. This is highlighted in Fig. 7a where the magnitude of the peak attributed to urate oxidation in $\mathrm{HB}$ was monitored over 14

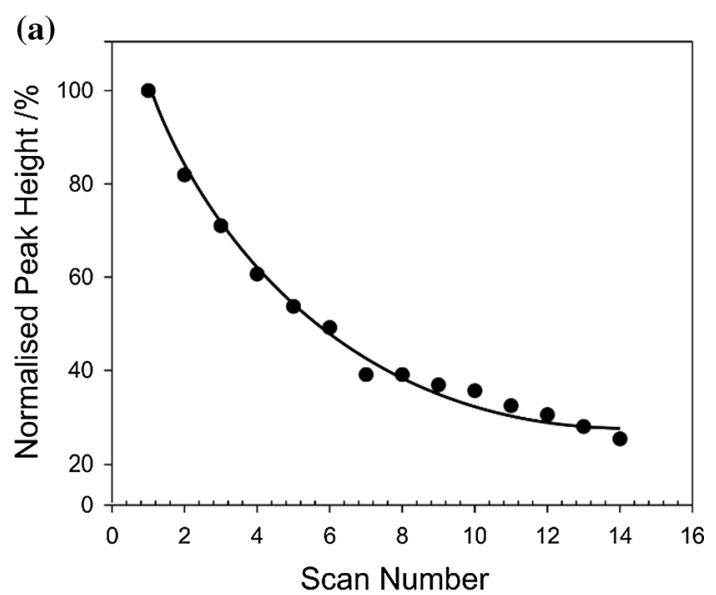

(b)

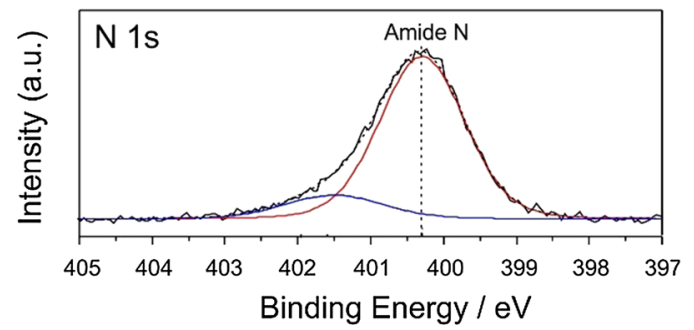

(c)

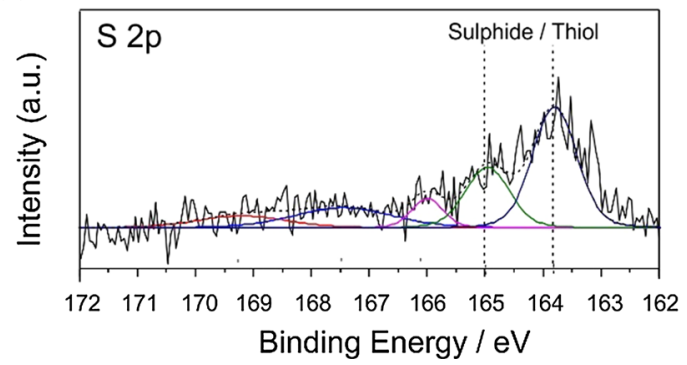

Figure 7 a Decrease in the urate oxidation peak height recorded at an anodised $\mathrm{MN}$ array in horse blood as a consequence of increasing scan number. XPS spectra detailing the $\mathrm{N} 1 \mathrm{~s}(\mathbf{b})$ and $\mathrm{S}$ $2 p$ (c) profiles as a result of the carbon-polystyrene composite being immersed in the blood sample for $30 \mathrm{~min}$. consecutive scans. There is a sustained decrease in the height with increasing scan number as the surface is progressively fouled. Surface contamination by proteinaceous material was confirmed through XPS analysis of the anodised carbon-polystyrene composites placed in HB. The $N$ 1s and $S 2 p$ spectral profiles obtained from an anodised carbon nanoparticle-polystyrene composite are detailed in Fig. 7b, c, respectively. While there is negligible $\mathrm{N}$ or $\mathrm{S}$ content in the anodised composite which had not been exposed to $\mathrm{HB}$, amide and thiol/disulphide functionalities were found on those that were immersed for $30 \mathrm{~min}$. It should be noted that similar profiles were observed with unanodised materials immersed for the same period with no significant difference in $\% \mathrm{~N}$ or $\% \mathrm{~S}$ content from that of the anodised variant. The presence of the amide $\mathrm{N}$ and thiol groups on the surface is consistent with peptide moieties that have accumulated at the electrode surface.

A complete analytical appraisal of the composite $\mathrm{MN}$ systems is beyond the scope of the present paper but it is clear from the results with horse blood that while they are able to respond to analytes within real/complex samples and perform as a conventional carbon electrode, fouling will be an issue-especially over long measurement periods. Surface fouling is an age old problem in electrochemistry and the $\mathrm{MN}$ systems are no different from other, generic, carbon electrodes. The application of the MN within transdermal applications will similarly encounter issues of fouling and, while this can be mitigated to some extent through conducting the analysis within a short time frame after insertion, permselective barriers (i.e., Nafion ${ }^{\circledR}$ ) may still be required where prolonged contact/periodic measurements are needed.

Leaving aside the issue of fouling, it is clear that the anodisation process enables the speedy creation of a range of oxygen functionality, which can dramatically improve the electroanalytical performance. While the interfacial composition is altered upon anodisation, electron microscopy revealed no appreciable change in the surface morphology from those shown in Fig. 2b. The detection of urate has received considerable attention and many electrode modification strategies have been pursued. Some of the more recent approaches are summarised in Table 2 along with the electrode performance indicators. While the electrochemical anodisation approach does not provide a detection limit which is at the forefront of sensing technology, it is nevertheless competitive 
Table 2 Electrode modifications employed in the detection of uric acid

\begin{tabular}{lllll}
\hline Electrode & Electrode modifier & LoD $(\mu \mathrm{M})$ & Sensitivity $\left(\mu \mathrm{Am}^{-1} \mathrm{~cm}^{-2}\right)$ & References \\
\hline $\mathrm{GCE}$ & Polyglycine/GR & 0.061 & & {$[36]$} \\
$\mathrm{Au} / \mathrm{IDA}$ & Herringbone CNT & 15 & & {$[37]$} \\
$\mathrm{CSPE}$ & Uricase ZnO QDs & 23 & 4.0 & {$[34]$} \\
$\mathrm{Al}$ & $\mathrm{MoS}_{2}$ & 1.17 & & {$[38]$} \\
$\mathrm{CSPE}$ & $\mathrm{GR} / \mathrm{Au} \mathrm{NPs}$ & 5.4 & & {$[39]$} \\
$\mathrm{GCE}$ & $\mathrm{ZnCl}_{2}$-CF & 0.11 & & {$[40]$} \\
$\mathrm{GCE}$ & $\mathrm{MoS}_{2} / \mathrm{rGO}$ & 0.74 & & {$[41]$} \\
$\mathrm{CF}$ & $\mathrm{Pt} \mathrm{NPs} / \mathrm{Au}-\mathrm{Sn}$ Alloy & 0.67 & 280 & {$[32]$} \\
$\mathrm{GCE}$ & $\mathrm{N} \mathrm{Doped} \mathrm{GR}_{\mathrm{GPE}}$ & 0.13 & 2064 & {$[33]$} \\
$\mathrm{GPE}$ & $\mathrm{XFO}_{3} \mathrm{NPs}(\mathrm{X}=\mathrm{La}, \mathrm{Ce}$ etc. $)$ & 0.2 & & {$[42]$} \\
$\mathrm{C}-\mathrm{PS} \mathrm{MN}$ & $\mathrm{TiO}_{2} \mathrm{NPs}$ & 0.07 & & {$[43]$} \\
\hline
\end{tabular}

GCE glassy carbon electrode, GPE graphite paste electrode, CSPE carbon screen-printed electrode, $C F$ carbon fibre; IDA interdigitated array, GR graphene, $r G O$ reduced graphene oxide, $C N T$ carbon nanotube, $Q D$ s quantum dots, NPs nanoparticles when considering the nature of the electrode fabrication and ease of modification. It should also be noted that many of the modifications in Table 2 would not be directly compatible with a transdermal application.

\section{Conclusions}

Microneedle based sensors have largely been based on metallic layers deposited onto the surface of prepatterned needle templates. The ability to create composite systems in which the formulation can be readily altered at low cost within conventional laboratory environments can however facilitate much more responsive development programmes. Moreover, the rich surface chemistry available at the carbon based systems through the simple processes of electrochemical anodisation is a critical advantage as it greatly increases the range of detection methodologies available to transdermal sensing. The minimally invasive merits of microneedle systems suggest considerable scope for biomedical sensing and the adoption of composite systems, as highlighted, here could open new avenues for electroanalytical approaches.

\section{Acknowledgements}

The authors are pleased to acknowledge financial support from the European Union's INTERREG VA Programme, managed by the Special EU Programmes
Body (SEUPB) and the Department for the Economy (DfE) Northern Ireland, the British Council (DSTUKIERI: Ref 65/2017) and the University of Central Lancashire Innovation and Enterprise for supporting this work.

\section{Authors Contributions}

$\mathrm{CH}$ was involved in design, manufacture and electrochemical characterisation of microneedles; SM and RJM contributed to XPS investigation, analysis and interpretation; RS was involved in guidance/analysis of carbon functionalities/protein adsorption; AM was involved in supervision and guidance on surface characterisation; JD contributed to supervision of overall project and compilation of manuscript.

Open Access This article is distributed under the terms of the Creative Commons Attribution 4.0 International License (http://creativecommons.org/ licenses/by/4.0/), which permits unrestricted use, distribution, and reproduction in any medium, provided you give appropriate credit to the original author(s) and the source, provide a link to the Creative Commons license, and indicate if changes were made.

\section{References}

[1] Rzhevskiy AS, Raghu T, Singh R, Donnelly RF, Anissimov YG (2017) Microneedles as the technique of drug delivery 
enhancement in diverse organs and tissues. J Control Release 2018(270):184-202

[2] Ita K (2017) Ceramic microneedles and hollow microneedles for transdermal drug delivery: two decades of research. J Drug Deliv Sci Technol 2018(44):314-322

[3] Donnelly RF, Larrañeta E (2018) Microarray patches: potentially useful delivery systems for long-acting nanosuspensions. Drug Discov Today 23(5):1026-1033

[4] Barrett C, Dawson K, Mahony CO, Riordan AO (2015) Development of low cost rapid fabrication of sharp polymer microneedles for in vivo glucose biosensing applications. ECS J Solid State Sci Technol 4(10):3053-3058

[5] Moga KA, Bickford LR, Geil RD, Dunn SS, Pandya AA, Wang Y et al (2013) Rapidly-dissolvable microneedle patches via a highly scalable and reproducible soft lithography approach. Adv Mater 36:5060-5066

[6] Ribet F (2018) Real-time intradermal continuous glucose monitoring using a minimally invasive microneedle-based system. Biomed Micromol 20:101

[7] Chiaranairungroj M, Pimpin A, Srituravanich W (2018) Fabrication of high-density microneedle masters towards the commercialisation of dissolving microneedles. Micro Nano Lett 13:284-288

[8] Sharma S, Huang Z, Rogers M, Boutelle M, Cass AEG (2016) Evaluation of a minimally invasive glucose biosensor for continuous tissue monitoring. Anal Bioanal Chem 29:8427-8435

[9] Sharma S, Saeed A, Johnson C, Gadegaard N, Eg A (2017) Sensing and bio-sensing research rapid, low cost prototyping of transdermal devices for personal healthcare monitoring. Sens Bio-Sens Res 13:104-108

[10] Lutton REM, Larrañeta E, Kearney M, Boyd P, Woolfson AD, Donnelly RF (2015) A novel scalable manufacturing process for the production of hydrogel-forming microneedle arrays. Int J Pharm 494(1):417-429

[11] Lim D, Vines JB, Park H, Lee S (2018) A versatile strategy for transdermal delivery of biological molecules. Int J Biol Macromol 110:30-38

[12] Zhu MW (2009) Silica needle template fabrication of metal hollow microneedle arrays. J Microeng 19:115010

[13] Cha K, Kim T, Park S, Kim D (2014) Simple and costeffective fabrication of solid biodegradable polymer microneedle arrays with adjustable aspect ratio for transdermal drug delivery using acupuncture microneedles. J Micromech Microeng 24:115015

[14] Bok K, Lee W, Cho C, Park D, Je S (2018) Chemical continuous glucose monitoring using a microneedle array sensor coupled with a wireless signal transmitter. Sens Actuators B Chem 2019(281):14-21
[15] Chinnadayyala SR, Park I, Cho S (2018) Nonenzymatic determination of glucose at near neutral $\mathrm{pH}$ values based on the use of nafion and platinum black coated microneedle electrode array. Microchim Acta 185:250

[16] McConville A, Davis J (2016) Transdermal microneedle sensor arrays based on palladium: polymer composites. Electrochem Commun 72:162-165. https://doi.org/10.1016/ j.elecom.2016.09.024

[17] Anderson A, Phair J, Benson J, Meenan B, Davis J (2014) Investigating the use of endogenous quinoid moieties on carbon fibre as means of developing micro $\mathrm{pH}$ sensors. Mater Sci Eng C. 43:533-537. https://doi.org/10.1016/j.ms ec.2014.07.038

[18] Desai D, Kumar A, Bose D, Datta M (2018) Biosensors and Bioelectronics Ultrasensitive sensor for detection of early stage chronic kidney disease in human. Biosens Bioelectron 105:90-94. https://doi.org/10.1016/j.bios.2018.01.031

[19] Brownlee BJ, Bahari M, Harb JN, Claussen JC, Iverson BD (2018) Electrochemical glucose sensors enhanced by methyl viologen and vertically aligned carbon nanotube channels. ACS Appl Mater Interfaces 10:28351-28360

[20] Dutt JSN, Cardosi MF, Livingstone C, Davis J (2005) Diagnostic implications of uric acid in electroanalytical measurements. Electroanalysis. 17(14):1233-1243

[21] Phair J, Newton L, McCormac C, Cardosi MF, Leslie R, Davis J (2011) A disposable sensor for point of care wound $\mathrm{pH}$ monitoring. Analyst 136(22):4692-4695

[22] Pratt IS (2018) Hypertension in children: role of obesity, simple carbohydrates, and uric acid. Front Public Health 6(May):1-7

[23] Kumagai T, Ota T, Tamura Y, Xiu W (2017) Time to target uric acid to retard CKD progression. Clin Exp Nephrol 21(2):182-192

[24] Zhong C, Zhong X, Xu T, Xu T, Zhang Y (2017) Sexspecific relationship between serum uric acid and risk of stroke: a dose-response meta-analysis of prospective studies. J Am Heart Assoc 6:e05042

[25] Kei A, Koutsouka F, Makri A, Elisaf M (2017) Uric acid and cardiovascular risk: what genes can say. Int J Clin Pract 2018:1-7

[26] El R, Tallima H (2017) Physiological functions and pathogenic potential of uric acid: a review. J Adv Res 8(5):487-493

[27] Lawal AT (2018) Progress in utilisation of graphene for electrochemical biosensors. Biosens Bioelectron 106(January):149-178

[28] Madhurantakam S, Babu KJ, Bosco J, Rayappan B (2018) Nanotechnology-based electrochemical detection strategies for hypertension markers. Biosens Bioelectron 116(February):67-80 
[29] Baig N, Rana A, Kawde A (2018) Modified electrodes for selective voltammetric detection of biomolecules. Electroanalysis 30(11):2551-2574

[30] Raymundo-Pereira PA, Baccarin M, Oliveira ON, Janegitz BC (2018) Thin Films and composites based on graphene for electrochemical detection of biologically-relevant molecules. Electroanalysis 30(9):1888-1896

[31] Azzouz A, Goud KY, Raza N, Ballesteros E, Lee S, Hong J et al (2019) Nanomaterial-based electrochemical sensors for the detection of neurochemicals in biological matrices. Trends Anal Chem 110:15-34

[32] Yang H, Zhao J, Qiu M, Sun P, Han D, Niu L et al (2018) Hierarchical bi-continuous $\mathrm{Pt}$ decorated nanoporous $\mathrm{Au}-\mathrm{Sn}$ alloy on carbon fiber paper for ascorbic acid, dopamine and uric acid simultaneous sensing. Biosens Bioelectron 2019(124-125):191-198

[33] Foroughi F, Rahsepar M, Kim H (2018) A highly sensitive and selective biosensor based on nitrogen-doped graphene for non-enzymatic detection of uric acid and dopamine at biological $\mathrm{pH}$ value. J Electroanal Chem 827(August):34-41

[34] Ali M, Shah I, Wan S, Sajid M, Hwan J, Hyun K (2018) Physical quantitative detection of uric acid through $\mathrm{ZnO}$ quantum dots based highly sensitive electrochemical biosensor. Sens Actuators A Phys 283:282-290

[35] Lilius E, Hyyppa S, Ps AR (1996) Accumulation of uric acid in plasma after repeated bouts of exercise in the horse. Comp Biochem Physiol Part B Biochem Mol Biol II(2):139-144

[36] He S, He P, Zhang X, Zhang X, Liu K, Jia L, Dong F (2018) Poly (glycine)/graphene oxide modified glassy carbon electrode: preparation, characterization and simultaneous electrochemical determination of dopamine, uric acid, guanine and adenine. Anal Chim Acta 1031:75-82

[37] Vidal L, Rodríguez-amaro R, Canals A, Morall E (2018) Evaluation of herringbone carbon nanotubes-modified electrodes for the simultaneous determination of ascorbic acid and uric acid. Electrochim Acta 285:284-291

[38] Sha R, Vishnu N, Badhulika S (2018) Chemical electrochemical sensor for highly selective detection of uric acid in human urine samples. Sens Actuators B Chem 2019(279):53-60

[39] Ji D, Liu Z, Liu L, Shin S, Lu Y, Yu X et al (2018) Smartphone-based integrated voltammetry system for simultaneous detection of ascorbic acid, dopamine, and uric acid with graphene and gold nanoparticles modified screenprinted electrodes. Biosens Bioelectron 119:55-62

[40] Zhang W, Liu L, Li Y, Wang D, Ma H, Ren H (2018) Electrochemical sensing platform based on the biomassderived microporous carbons for simultaneous determination of ascorbic acid, dopamine, and uric acid. Biosens Bioelectron 121(August):96-103

[41] Zhao Y, Zhou J, Jia Z, Huo D, Liu Q, Zhong D et al (2019) In-situ growth of gold nanoparticles on a 3D-network consisting of a MoS2/rGO nanocomposite for simultaneous voltammetric determination of ascorbic acid, dopamine and uric acid. Mikrochim Acta 186:92

[42] Kumar Y, Pradhan S, Pramanik S, Bandyopadhyay R (2018) Efficient electrochemical detection of guanine, uric acid and their mixture by composite of nano-particles of lanthanides ortho-ferrite XFeO 3 (X = La, Gd, Pr, Dy, Sm, Ce and Tb). J Electroanal Chem 830-831:95-105

[43] Biswas S, Pradhan S, Naskar H, Bandyopadhyay R, Pramanik P (2018) Sol-gel synthesis of cubic titanium dioxide nanoparticle using poly (ethylene glycol) as a capping agent: voltammetric simultaneous determination of uric acid and guanine. Microchim Acta 185(11):513

Publisher's Note Springer Nature remains neutral with regard to jurisdictional claims in published maps and institutional affiliations. 